\title{
Asymmetric Functionalization of Gold Nanoparticles with Oligonucleotides
}

\author{
Xiaoyang Xu, Nathaniel L. Rosi, Yuhuang Wang, Fengwei Huo, and Chad A. Mirkin ${ }^{*}$ \\ Department of Chemistry and International Institute for Nanotechnology, Northwestern University, \\ 2145 Sheridan Road, Evanston, Illinois 60208
}

Programmable assembly methods based upon the use of oligonucleotide-functionalized nanoparticles and sequence-specific assembly with complementary DNA have led to the development of a variety of fundamentally interesting materials and technologically significant detection systems. ${ }^{1-3}$ The attractive feature of this approach to materials synthesis is that one can control the size, shape, and compositions of the individual nanoparticle building blocks as well as their spacing and periodicity within a macroscopic and, often times, polymeric structure through judicious choice of nanoparticle building block and DNA linkers. Most of the work in this area has focused on the use of isotropically functionalized particles since there are very few ways of selectively functionalizing different surface regions of an individual particle. However, if one could deliberately functionalize only one hemisphere or one distinct point on a particle in a general way, one could begin to introduce valency into such structures, thereby allowing greater control over the assembly process. ${ }^{4}$

A kinetic control approach, developed by Alivisatos and coworkers, allows one to functionalize nanoparticles with as few as one oligonucleotide per particle. ${ }^{2 b, 5}$ This novel strategy introduces anisotropy into such particles and has enabled the assembly of dimer and trimer structures not attainable with the isotropically functionalized particles. Although this was an important step forward in nanoparticle functionalization, it has been limited to very small particles and typically leads to mixtures of products that must be separated by electrophoretic means. Here, we report a general strategy to functionalize a AuNP with two different types of oligonucleotides in a site-specific manner by using a magnetic sphere as a geometric restriction template (Scheme 1).

Anisotropic functionalization of AuNPs was accomplished using a three-component assembly strategy (Scheme 1) consisting of the following: (1) magnetic microparticles (MMPs, $2.8 \mu \mathrm{m}$ diameter polystyrene particles with iron oxide cores) functionalized with 3 'thiol-terminated 30-mer oligonucleotides 1, (2) 3'-hydroxyl-modified "extension" oligonucleotides $\mathbf{2}$ that are complementary to half of the MMP oligonucleotides, and (3) AuNPs (13 nm, citrate-stabilized particles) densely functionalized with 3 '-thiolated and 5'-phosphorylated 15-mer oligonucleotides 3 that are half-complementary to the other half of the MMP oligonucleotides. Standard methods were used to functionalize the MMPs and AuNPs with oligonucleotides (see Supporting Information). After combining the three components in the presence of a ligation buffer, they assembled to form complex $\mathbf{4}$ in which the oligonucleotide-modified MMP acts as a template to co-align the 3'-hydroxy group of the extension oligonucleotides with the 5'phosphate group of the AuNP oligonucleotide. T4 DNA ligase was added to the reaction solution to catalyze the formation of a phosphodiester bond between the $3^{\prime}$-hydroxyl and the 5 '-phosphate of the extension oligonucleotides and the oligonucleotide chemisorbed to the AuNP, respectively, thus affording new 30-mer oligonucleotides bound only to the sections of

E-mail: chadnano@northwestern.edu. 
the AuNP that could hybridize with the MMP template. ${ }^{6}$ After ligation, resulting complex 5 was removed from the reaction mixture via magnetic separation, washed with ligation buffer, and the new anisotropically modified AuNPs 6 were released from the MMP templates by heating.

DNA melting experiments provide a definitive means of tracking the enzymatic ligation step. The AuNPs can be reversibly released from the MMPs by raising the temperature above the melting point of the duplex DNA linkers. By monitoring extinction at $520 \mathrm{~nm}$ (the plasmon resonance for the AuNPs) as a function of temperature, the melting point, $T_{\mathrm{m}}$, of the duplex DNA linkers after ligation was determined to be $74.5^{\circ} \mathrm{C}$, approximately $20{ }^{\circ} \mathrm{C}$ above the $T_{\mathrm{m}}$ duplex before ligation (Figure 1A, compare blue and red lines). This significant increase in $T_{\mathrm{m}}$ is consistent with conversion of the three-strand $30 \mathrm{bp}$ nicked structure to a continuous twostrand $30 \mathrm{bp}$ duplex. SEM analysis of the particles before melting shows that thousands of particles are attached to each MMP (Figure 1B). Importantly, after the ligation step, the melting process is characterized by a single sharp melting transition, indicating that all of the AuNPs hybridized on the surfaces of the MMPs were ligated to the extension oligonucleotides.

Isolation of the ligated AuNPs from the MMPs was achieved by dehybridization in nanopure water followed by removal of the MMPs with a magnetic separator. The extended strands introduce asymmetry into the surface structure of the DNA-functionalized AuNP, which should allow one to program particle assembly via hybridization in a highly directional manner. As a proof-of-concept, we mixed 13 and $30 \mathrm{~nm}$ AuNPs (molar ratio 10:1), both of which were asymmetrically functionalized with complementary extension oligonucleotides. Because only the extension oligonucleotides of the AuNPs can hybridize, a "cat paw" structure was formed (Figure 2A,B and Figure S3 Supporting Information). The cat paw structures suggest that, for each $30 \mathrm{~nm}$ AuNP, approximately $1 / 3$ to $1 / 2$ of its surface is asymmetrically functionalized with the extension oligonucleotides.

To further demonstrate the capabilities of this assembly strategy, we reacted asymmetrically functionalized $13 \mathrm{~nm}$ AuNPs with $30 \mathrm{~nm}$ AuNPs that were functionalized with oligonucleotides complementary only to the extended strands on the $13 \mathrm{~nm}$ AuNP. Because of the asymmetric functionalization of AuNP 6, the two sets of nanoparticles did not aggregate, but instead formed satellite structures consisting of one $30 \mathrm{~nm}$ AuNP surrounded by $13 \mathrm{~nm}$ AuNPs. TEM analysis of the sample reveals that nearly every $30 \mathrm{~nm}$ AuNP was hybridized with six to ten $13 \mathrm{~nm}$ AuNPs (Figure 1C,D). Although drying of the sample and the electron beam can substantially affect the state of the DNA duplex interconnects, the TEM data do conclusively show that the asymmetry of $\mathbf{6}$ stops the sequence-specific oligomerization process in the form of the satellite structure. Notably, the satellite-like nanoparticle complexes exhibit a $6 \mathrm{~nm}$ red shift in their surface plasmon absorption compared with what is normally observed for dispersed $30 \mathrm{~nm}$ AuNPs (Figure S1, Supporting Information). This red shift is consistent with Mie theory and the formation of a small aggregate as opposed to the large polymeric structures typically attained with the isotropically functionalized particles. ${ }^{7}$ Dynamic light scattering measurements also confirm the formation of satellite structures with an average diameter of $152 \pm 10 \mathrm{~nm}$, which is approximately the diameter one would expect from modeling the satellite structure made from the two different sizes of AuNP building blocks and DNA interconnects (Figure S2, Supporting Information).

A third type of nanostructure that resembles a dendrimer is possible through these asymmetrically functionalized particles. For example, we have prepared satellite structures from 30 and $60 \mathrm{~nm}$ AuNPs that resemble those in Figures 2C. The dendrimer-like structures are formed by further hybridizing the nonextended oligonucleotides on the $30 \mathrm{~nm}$ particles with complementary extension oligonucleotides on asymmetrically functionalized $13 \mathrm{~nm}$ AuNPs (Figure 2E,F and Figure S4 Supporting Information). This three-component structure 
demonstrates that this method and asymmetrically functionalized particles can be used to precisely control the assembly of at least three different AuNPs into designed heterostructures in a step-by-step fashion.

In conclusion, we have demonstrated a strategy for asymmetric functionalization of AuNPs with oligonucleotides. The DNA-functionalized MMPs serve a dual-purpose: they allow sitespecific modification of DNA-modified AuNPs, and they facilitate the separation and purification of the anisotropically functionalized AuNPs. AuNPs functionalized in this way exhibit highly directional selectivity for hybridization with other nanoparticles, allowing the design and assembly of unique nanoparticle heterostructures, such as satellite, cat paw, and dendrimer-like structures. This is a significant step toward building valency into nanoparticle building blocks that allow for the deliberate design and synthesis of sophisticated nanostructured materials.

\section{Supplementary Material}

Refer to Web version on PubMed Central for supplementary material.

\section{Acknowledgements}

This work was supported by the AFOSR, DARPA, NSF, and a NIH Director's Pioneer Award.

\section{References}

1. (a) Mirkin CA, Letsinger RL, Mucic RC, Storhoff JJ. Nature 1996;382:607-609. [PubMed: 8757129] (b) Mucic RC, Storhoff JJ, Mirkin CA, Letsinger RL. J Am Chem Soc 1998;120:12674-12675. (c) Gothelf KV, LaBean TH. Org Biomol Chem 2005;3:4023-4037. [PubMed: 16267576] (d) Katz E, Willner I. Angew Chem, Int Ed 2004;43:6042-108. (e) Niemeyer CM, Ceyhan B, Hazarika P. Angew Chem, Int Ed 2003;42:5766-5770. (f) Bates AD, Callen BP, Cooper JM, Cosstick R, Glidle A, Jaeger L, Pearson JL, Proupin-Perez M, Xu C, Cumming DRS. Nano Lett 2006;6:445-448. [PubMed: 16522039] (g) Le JD, Pinto Y, Seeman NC, Musier-Forsyth K, Taton TA, Kiehl RA. Nano Lett 2004;4:2343-2347. (h) Zhang J, Liu Y, Ke Y, Yan H. Nano Lett 2006;6:248-251. [PubMed: 16464044] (i) Mbindyo JKN, Reiss BD, Martin BR, Keating CD, Natan MJ, Mallouk TE. Adv Mater 2001:249-254. (j) Cobbe S, Connolly S, Ryan D, Nagle L, Eritja R, Fitzmaurice D. J Phys Chem B 2003;107:470-477. (k) Sadasivan S, Dujardin E, Li M, Johnson CJ, Mann S. Small 2005:103-106. [PubMed: 17193359]

2. (a) Alivisatos AP, Johnsson KP, Peng X, Wilson TE, Loweth CJ, Bruchez MP Jr, Schultz PG. Nature 1996;382:609-611. [PubMed: 8757130] (b) Loweth CJ, Caldwell WB, Peng X, Alivisatos AP, Schultz PG. Angew Chem, Int Ed 1999;38:1808-1812.

3. Rosi NL, Mirkin CA. Chem Rev 2005;105:1547-1562. [PubMed: 15826019]

4. Glotzer SC. Science 2004;306:419-420. [PubMed: 15486279]

5. Zanchet D, Micheel CM, Parak WJ, Gerion D, Williams SC, Alivisatos AP. J Phys Chem B 2002;106:11758-11763.

6. Kanaras AG, Wang Z, Bates AD, Cosstick R, Brust M. Angew Chem, Int Ed 2003;42:191-194.

7. Lazarides AA, Schatz GC. J Phys Chem B 2000;104:460-467. 

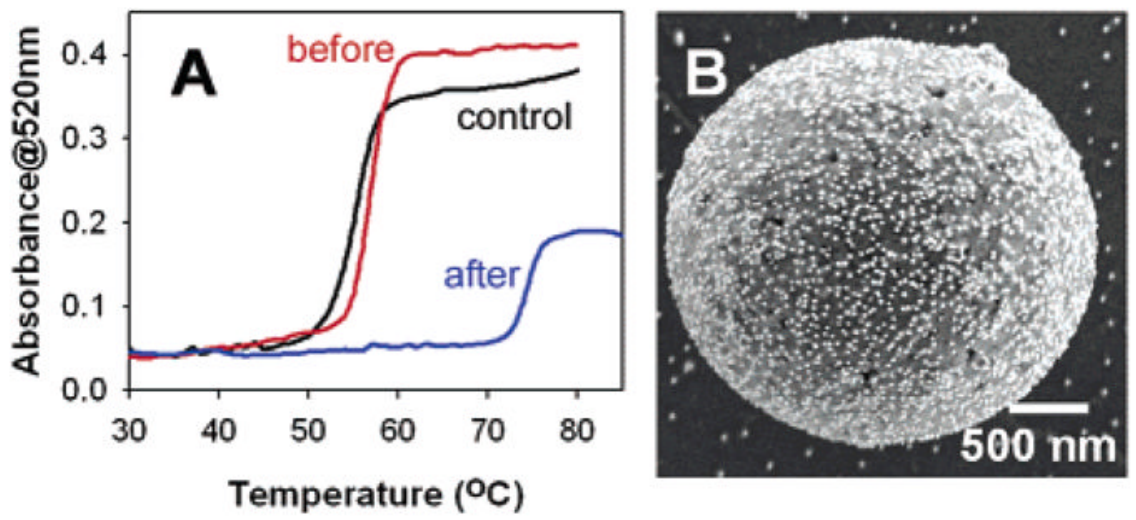

Figure 1.

(a) DNA melting curves of $13 \mathrm{~nm}$ AuNPs that are hybridized with MMPs through an extension DNA. The ligation step significantly increased the melting temperature, from $54{ }^{\circ} \mathrm{C}$, before (red), to $74.5^{\circ} \mathrm{C}$, after (blue). Without the extension DNA, the ligation step showed minimal effect (black curve). (b) SEM image showing $30 \mathrm{~nm}$ AuNPs on the surface of a MMP. 


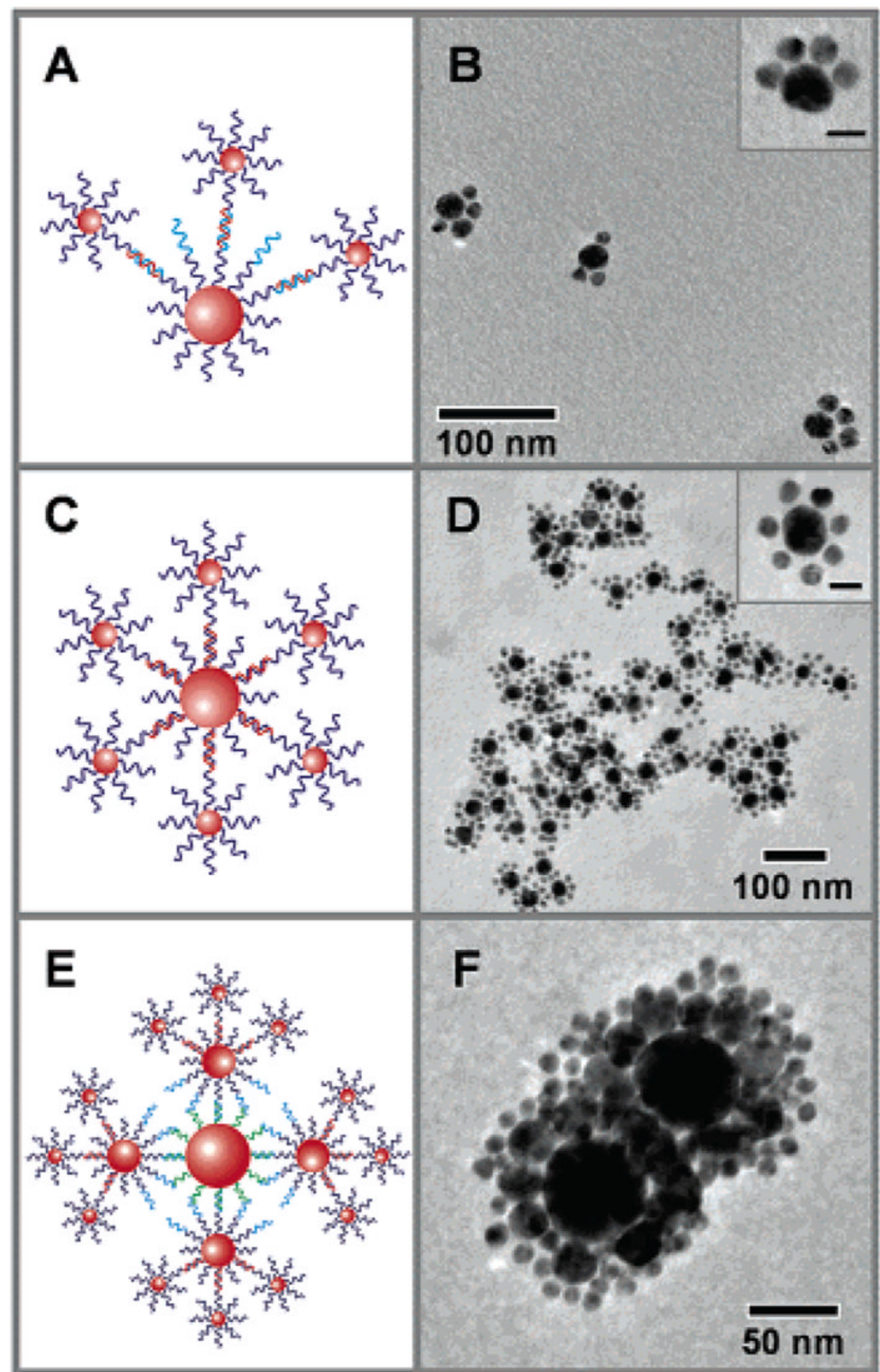

Figure 2.

Directional assembly of asymmetrically functionalized AuNPs into (A, B) cat paw, (C, D) satellite, and (E, F) dendrimer-like structures. Inset: scale bar $=20 \mathrm{~nm}$. 

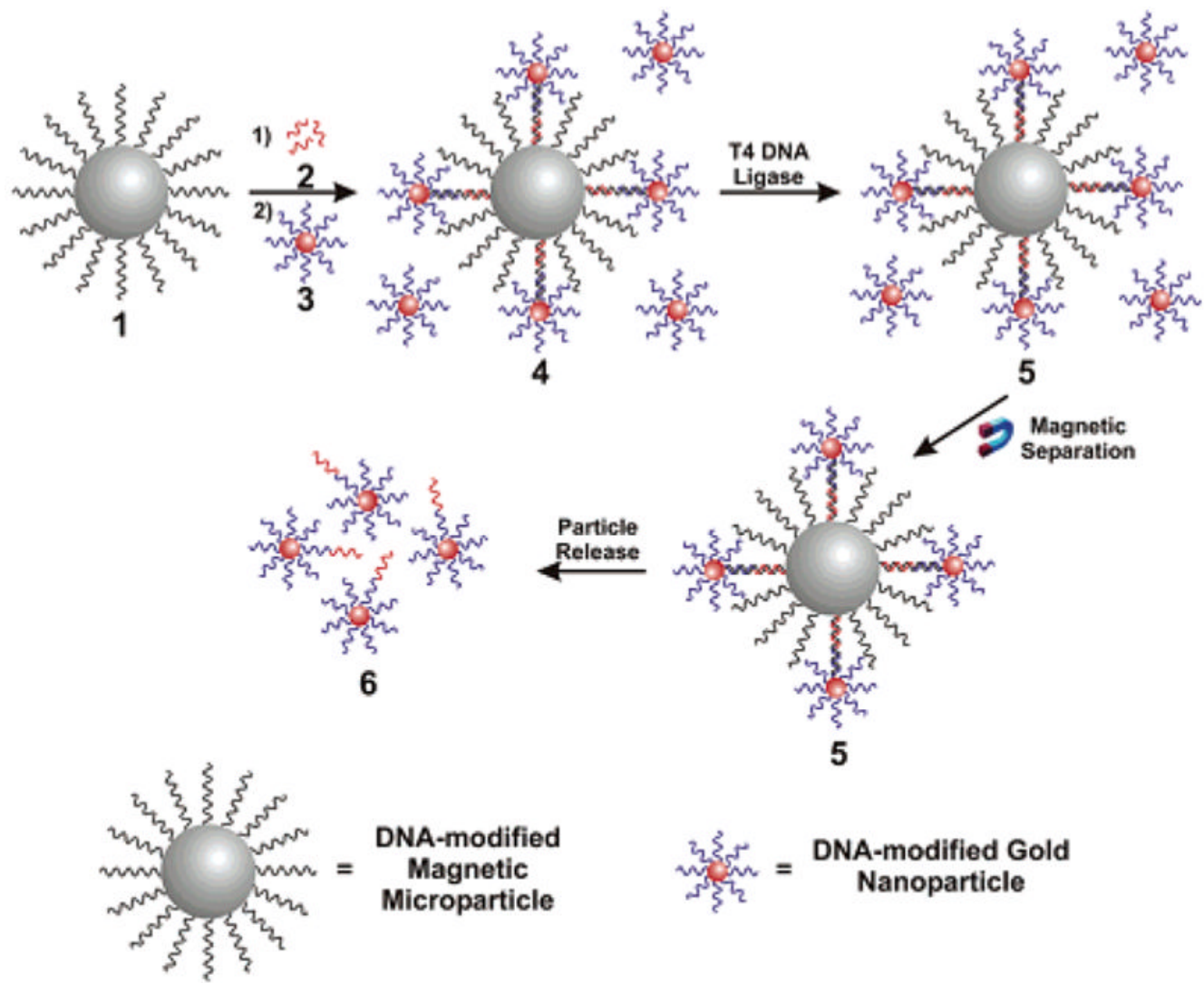

Scheme 1a.

${ }^{a}$ Note that we are not intending to imply that particle 6 has a single extended linker, but rather a small collection of extended oligonucleotides localized in one region. 